\title{
Effect of Topical Grape Sap on Apoptosis in Hair Follicle Among Male
}

\section{Rats}

\author{
Zahra Esmaeilzadeh ${ }^{1}$, Ahmad Shabanizadeh ${ }^{2}$, Zahra Taghipour ${ }^{1}$, Reza Vazirinejad ${ }^{3}$, Mohammad Reza \\ Salahshoor ${ }^{4}$ and Mohammad Mohsen Taghavi ${ }^{5,{ }^{*}}$ \\ ${ }^{1}$ Department of Anatomical Sciences, Faculty of Medicine, Rafsanjan University of Medical Sciences, Rafsanjan, Iran \\ ${ }^{2}$ Department of Anatomical Sciences, Immunology of Infectious Disease Research Center, Research Institute of Basic Medical Sciences, Faculty of Medicine, Rafsanjan \\ University of Medical Sciences, Rafsanjan, Iran \\ ${ }^{3}$ Department of Social Medicine, Social Determinants of Health Research Center, Faculty of Medicine, Rafsanjan University of Medical Sciences, Rafsanjan, Iran \\ ${ }^{4}$ Department of Anatomical Sciences, Faculty of Medicine, Kermanshah University of Medical Science, Kermanshah, Iran \\ ${ }^{5}$ Department of Anatomical Sciences, Social Determinants of Health Research Center, Faculty of Medicine, Rafsanjan University of Medical Sciences, Rafsanjan, Iran \\ "Corresponding author: Department of Anatomical Sciences, Social Determinants of Health Research Center, Faculty of Medicine, Rafsanjan University of Medical Sciences, \\ Rafsanjan, Iran. Email: taghavi164@yahoo.com
}

Received 2021 April 27; Revised 2021 July 06; Accepted 2021 July 31.

\begin{abstract}
Background: Hair loss is an emotional and stressful condition with an unpredictable profound impact on the social interactions of patients.

Objectives: This study aimed to evaluate the effect of grape sap on apoptosis in hair follicles.

Methods: This experimental study was performed on 126 male Wistar rats within a weight range of $30 \pm 250$ g. The rats were assigned into seven groups, namely bleomycin group, normal saline group, grape sap group (1 mg/kg), grape sap group (10 mg/kg), grape sap group (100 mg/kg), minoxidil group, and minoxidil plus grape sap group (100 mg/kg). The rats received bleomycin (1.7 $\mathrm{mL} / \mathrm{kg}$, four times with the interval of 5 days) and then were treated with grape sap for 21 days. The skin samples were taken from rats on days 7, 14, and 21 (i.e., the last day of the treatment).

Results: The results showed a significant increase in the groups treated with grape sap, compared to the bleomycin-treated group in terms of the number of follicles, sebaceous glands, and blood vessels at the base of every follicle, hair growth length, total antioxidant capacity, and BCL2 gene expression. The use of grape sap showed beneficial effects on the reduction of hair fall.

Conclusions: According to the results, it seems that grape sap can be employed as a non-chemical drug due to its rich compounds, especially antioxidants, and decreases apoptosis in hair follicle cells through increasing the expression ratio of $B C L 2 \mid B A X$, thereby stimulating hair growth.
\end{abstract}

Keywords: BAX, BCL2, Grape Sap, Hair Follicle, Rat

\section{Background}

Hair is regarded as one of the most important parts of the human body. It also plays a crucial role in providing insulation to the body, a sense of beauty, and self-confidence in the individuals $(1,2)$. Despite multiple drug therapies, many individuals still suffer from hair loss or slow hair growth (3). Hair loss can be related to emotional stress or anxiety in humans (4).

Although dead or dormant hair follicles in humans are not considered traumatic events, they have a profound impact on the social interactions and mental health of patients $(5,6)$. Moreover, hair loss is one of the most important problems that psychologically affect cancer patients undergoing chemotherapy. This disorder may also hinder their treatment process (7-9). Therefore, it is essential to provide the patients with a treatment that can prevent further hair loss with fewer side effects $(10,11)$.

There are different combinations (e.g., minoxidil and finasteride) that are currently utilized for hair loss treatment; however, the aforementioned combination therapy has been reported ineffective due to severe side effects and non-persistent positive effects after stopping the medication $(12,13)$. In recent years, herbal medicines have been used in most countries and have replaced conventional medical treatments in many cases (14). The importance of focusing on complementary medicine is crucial due to the severe side effects of current hair loss treatments. Therefore, it is essential to present medicinal herbs which prevent hair loss and encourage new hair growth without 
drug-related side effects (15-17).

Grape sap is a liquid that flows in early spring when the grapevine branches are severed. This liquid has antioxidant and anti-inflammatory properties and contains water, glucose, fructose, calcium, potassium, iron, magnesium, phosphate, tartaric acid, citric acid, fumaric acid, malic acid, succinic acid, vitamin B1, vitamin B6, glutamic acid, glutamine, proline, and alanine (18). With this background in mind, this study aimed to evaluate the effect of grape sap on apoptosis in the hair follicles of rats.

\section{Methods}

With regard to the standard ethical guidelines (National Institutes of Health, publication no. $85-23$, revised 1985; European Communities Directive 86/609/EEC) and approved by the Local Ethics Committee of Rafsanjan University of Medical Sciences, 126 male Wistar rats within the weight range of $30 \pm 250 \mathrm{~g}$ were acquired from the Animal House of Rafsanjan University of Medical Sciences, Kerman, Iran. The rats were fed with sufficient water and food and kept under standard laboratory conditions (12: 12 h/light-dark cycle at $20 \pm 2^{\circ} \mathrm{C}$ and $52 \%$ air humidity) in the animal house of Rafsanjan University of Medical Sciences.

Bleomycin $(1.7 \mathrm{~mL} / \mathrm{kg}$, four times with the interval of 5 days) was intraperitoneally injected into the rats under study. This anticancer agent causes animal hairs to enter the telogen phase, in which hair starts to fall out or gets ready to fall out. In either case, hair is not in the anagen phase (19). Subsequently, the rats were randomly (i.e., giving each rat a number and then using a random number table) assigned to seven groups with equal numbers $(\mathrm{n}=$ 18) as follows:

- Bleomycin-treated group: Bleomycin was intraperitoneally injected $(1.7 \mathrm{~mL} / \mathrm{kg}$, four times with an interval of 5 days) to cause hair loss in this group. The rats did not receive anything after taking bleomycin.

- Normal saline plus Bleomycin-treated group: Normal saline was completely applied to the entire skin surface (i.e., back of the head, the area between two ears).

- Grape sap-treated group: One milligram of the grape sap extraction was subcutaneously injected per kilogram of the rat body weight $(1 \mathrm{mg} / \mathrm{kg})$.

- Grape sap-treated group (10 mg/kg).

- Grape sap-treated group (100 mg/kg).

After the last administration of bleomycin $(2 \mathrm{~mL})$ to the rats, the grape sap with the aforementioned doses was topically applied to the affected areas in groups 3,4 , and 5 once daily for 21 days.

- Minoxidil-treated group: After the last administration of bleomycin $(2 \mathrm{~mL})$ to the rats, the minoxidil lotion ) 5 H15N5O) was applied to the target areas for 21 days.
- Minoxidil plus grape sap-treated group (100 mg/kg).

After the last administration of bleomycin $(2 \mathrm{~mL})$ to the rats, minoxidil plus grape sap $(100 \mathrm{mg} / \mathrm{kg})$ was topically applied to the target areas.

The rats were anesthetized with ether after the last administration of bleomycin, and the skin samples were collected from the back of the rats' heads on days 7, 14, and 21. The specimens obtained from all seven groups were divided into two halves to evaluate the number and length of hair follicle growth, number of sebaceous glands and blood vessels at the base of every follicle, and expression of $B A X$ and $B C L 2$ genes (20).

Half of the samples were initially fixed and passaged for histological studies. The paraffin blocks were prepared and sectioned ( $5 \mu \mathrm{m}$ thick) using a rotary microtome. Furthermore, 10 slides of each sample were randomly selected and stained with hematoxylin-eosin (H\&E) and periodic acid-Schiff(PAS).

The second half of the samples were utilized for molecular diagnostics. First, the skin samples were immediately transferred to nitrogen tanks to check the real-time. After collecting the skin samples of all groups using a homogenizer, the ribonucleic acid (RNA) of the animal skins was extracted under sterile homogeneous conditions to make complementary deoxyribonucleic acid (cDNA). An RNA Extraction Kit (catalog no.: A101231; Pars Tous Co., Iran) and subsequently Easy cDNA Kit (catalog no.: A101161; Pars Tous Co., Iran) were used to extract RNA and cDNA (Table 1). The blood samples were also centrifuged (3000 rpm) for $5 \mathrm{~min}$ to separate serum and stored at $-20^{\circ} \mathrm{C}$ until the initiation of the experiments.

The grape sap of Shahani grapevines was collected in Rabor, Kerman, Iran, and placed in a freeze dryer for 48 $\mathrm{h}$ to generate powder extracts. Microscopic sections were prepared and stained for histopathology. The images (1500 $\mu \mathrm{m})$ of the sections of the skin surface were then taken using a conventional Olympus microscope (DP25-BX51) and Cell A software $(\times 40, \times 20$, and $\times 10$ magnification). The quantitative data (mean \pm standard deviation) were analyzed in SPSS software (version 18) through analysis of variance and Tukey's test to compare the measured variables between different groups. A P-value of less than 0.05 was considered statistically significant.

\section{Results}

The hair follicle glassy basement membranes were evaluated utilizing PAS staining. According to the results, there were no significant differences between the treatment groups and the control group regarding the thickness of the glassy basement membranes. The H\&E staining was used to evaluate healthy hair follicles parameters, 


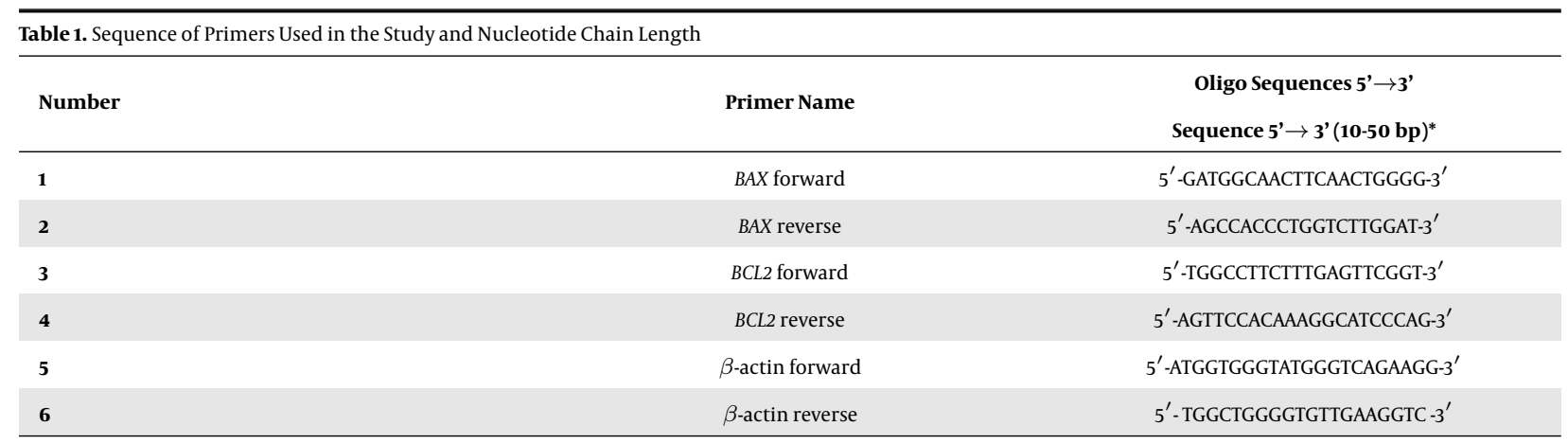

such as the rounded nucleus, prominent nucleolus, uniform and bright chromatin pattern, and certain size limits. Moreover, telogenic hair follicles were measured using the parameters, including wrinkled and high-density nuclei and obscure nucleoli.

Regarding the bleomycin-treated group, most hair follicles were in the telogen phase with wrinkled and highdensity nuclei and obscure nucleoli (Figure 1C). The results obtained from the normal saline group indicated healthy follicles with rounded nuclei, prominent nucleolus, and follicles with a certain size limit (Figure 1D). Three groups were treated with three different doses of the grape sap (i.e., 1, 10, and $100 \mathrm{mg} / \mathrm{kg}$ ) (Figures $1 \mathrm{~A}$ and $\mathrm{B}$ ). The follicles of these groups were healthy with rounded nuclei, prominent nucleolus, a certain size limit, and integrated tissue. Moreover, several hair follicles were observed in the telogen phase. The number of hair follicles in the telogen phase was higher in the grape sap group with a dose of $1 \mathrm{mg} / \mathrm{kg}$, compared to those reported for the groups with doses of 10 and $100 \mathrm{mg} / \mathrm{kg}$.

Concerning the minoxidil-treated group, the follicles had rounded nuclei, prominent nucleolus, a certain size limit, and integrated tissues. In the minoxidil plus grape sap (100 mg/kg) group, the follicles had rounded nuclei, prominent nucleolus, a certain size limit, and integrated tissues. The number of hair follicles and sebaceous glands, hair growth length, and blood vessel density at the base of every follicle of the hair were evaluated in this study in seven experimental groups.

According to the results, there were significant differences between the grape sap-treated groups and the bleomycin-treated group in terms of the number of anagenic hair follicles (Figure 2), number of sebaceous glands, vascular density, and length of hair growth. The results obtained from the grape sap-treated groups were quite similar to those reported for the minoxidil-treated group. According to the results of the total antioxidant capacity analysis, there was a significant increase in the grape saptreated group (100 mg/kg) and minoxidil plus grape sap
(100 $\mathrm{mg} / \mathrm{kg}$ )-treated group on day 21 , compared to that reported for the bleomycin-treated group (Figure 3).

According to the results of real-time polymerase chain reaction, a significant increase was observed in terms of $B C L 2$ expression in the grape sap-treated group $(100 \mathrm{mg} / \mathrm{kg})$ on day 21, compared to that reported for other treated groups (Figure 4; $\mathrm{P}<0.001$ ). The obtained results of this study showed the positive effects of grape sap on the prevention of hair loss. There was an increase in the number of follicles in the anagen phase, sebaceous glands, hair growth length, blood vessel density at the base of every hair follicle, and expression of BCL2. However, a decrease was observed in $B A X$ expression within the treated groups (Figure 5).

\section{Discussion}

In recent years, herbal medicines have been used in most countries and have replaced conventional medical treatments in many cases. Focusing on complementary medicine is crucial due to the severe side effects of current hair loss treatments. A study performed by Yoon et al. showed that the topical application of jujube oil on the back of 15 mice increased longitudinal growth and hair thickness (21).

Takahashi et al. conducted a study using the nanoemulsion technique in 1999. The results showed that grape seed extract with tocopherol reduced oxidative stress leading to hair growth increase (22). Similarly, the findings of the present study showed an increase in hair growth; however, different techniques and doses of grape sap were employed in the current study. In a study performed by Li et al., the effect of Chrysanthemum extract on hair growth and hair follicle proliferation was evaluated among C57BL/6 mice. Immunohistochemistry results revealed the positive effect of chrysanthemum extract on repairing damaged hair follicles due to containing the antioxidant compounds resulting in hair growth increase and hair follicle proliferation (23). The obtained results 

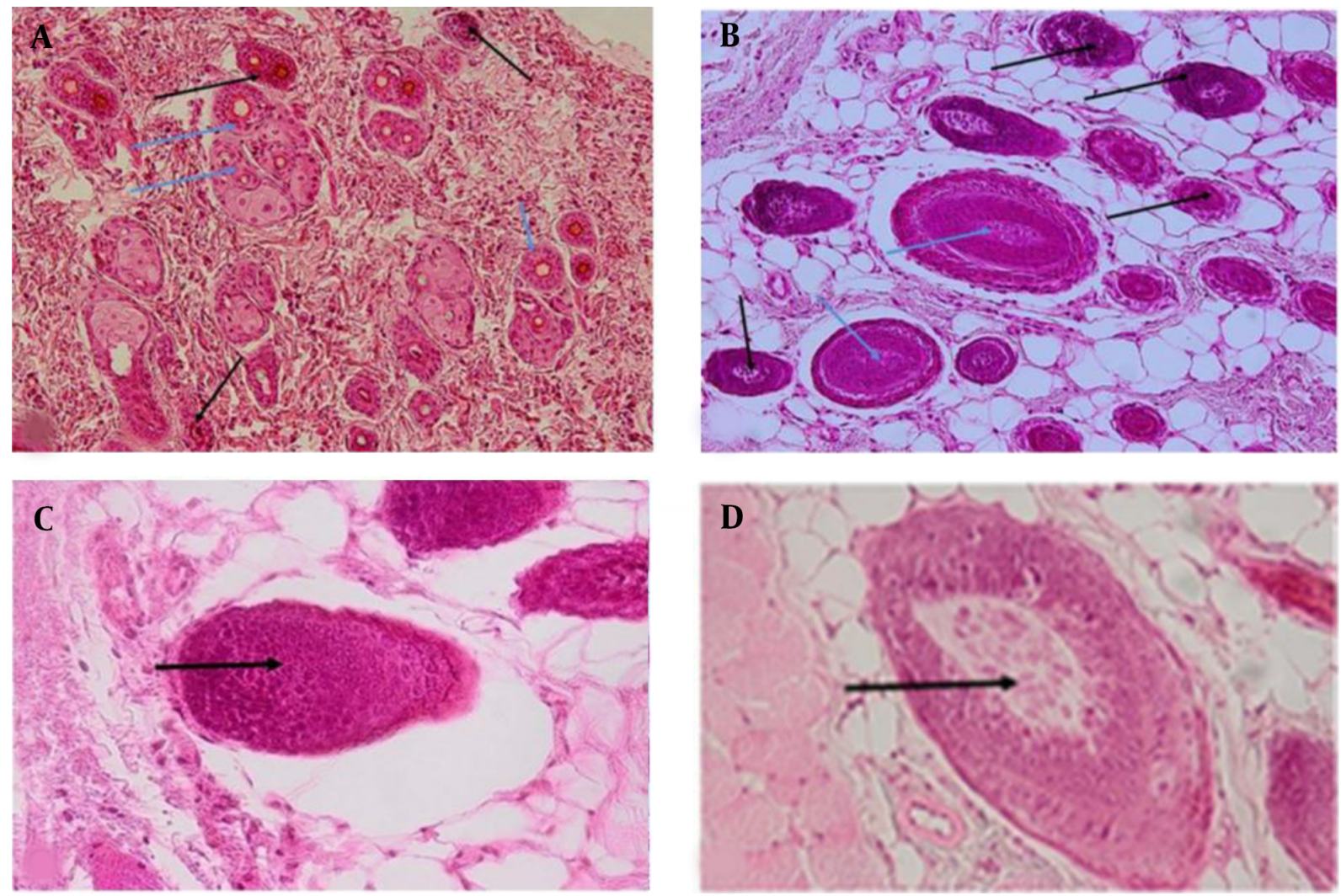

Figure 1. Hematoxylin-eosin staining in experimental groups 21 days after the last administration; Black and white arrows showing the telogenic and anagenic hair follicles, respectively; A, Grape sap-treated group (10 mg/kg); B, Grape sap-treated group (100 mg/kg); C, untreated group receiving bleomycin 21 days after the last administration $(\times 20$ magnification), arrow showing the telogenic hair follicle; D, Normal saline group 21 days after the last administration $(\times 20$ magnification), arrow showing the anagenic hair follicle $(\times 10$ magnification).

of the current study are consistent with the results of the aforementioned study. However, grape sap was used in this study which revealed an increase in histomorphological factors.

Moreover, Singha et al. (2015) investigated the antioxidant effects of four different grapevine extracts on human lymphocyte cells. According to the results, the grapevine extract was regarded as a potential source of natural antioxidants resistant to oxidative stress caused by ionizing radiation. In addition, the extracts were effective in the inhibition of apoptosis (24). The obtained results of the total antioxidant analysis in the grape sap-treated groups are consistent with the findings of the aforementioned study.

In a study carried out by Patel et al. (2015), it was reported that medicinal plants, such as Phyllanthus emblica, Fenugreek, and Buxus sempervirens containing epigallocatechin-3-gallate, procyanidin B2, and B3, and proanthocyanidin, could prevent hair loss. Moreover, they increased hair growth through the enhancement of circulation and hair follicle volume and prolongation of the an- agen phase (25). The obtained results of the present study are in line with the results of the aforementioned study.

There was an increase in blood vessel density at the base of every hair follicle and anagenic follicles in grape sap-treated groups, compared to those of other groups in the present study. Furthemore, Koparal and Bostancioglu (2016) showed that the seed extract of Delphinium exaltatum improved hair growth by increasing the expression of angiogenesis factor (26). The results obtained from the groups with higher antioxidant absorption are in line with the results of the aforementioned study. The results of the present study showed a significant increase in the antioxidant absorption coefficient. Furthermore, the grape sap increased the histomorphological factors, which may be due to the expression of angiogenesis factors.

A study conducted by Zhong et al. investigated the effect of grape seed procyanidin B2 on hepatocytes. The results showed that procyanidin reduced oxidative stress, which led to the reduction of apoptosis (27). In the current study, there was a reduction in apoptosis and an increase 


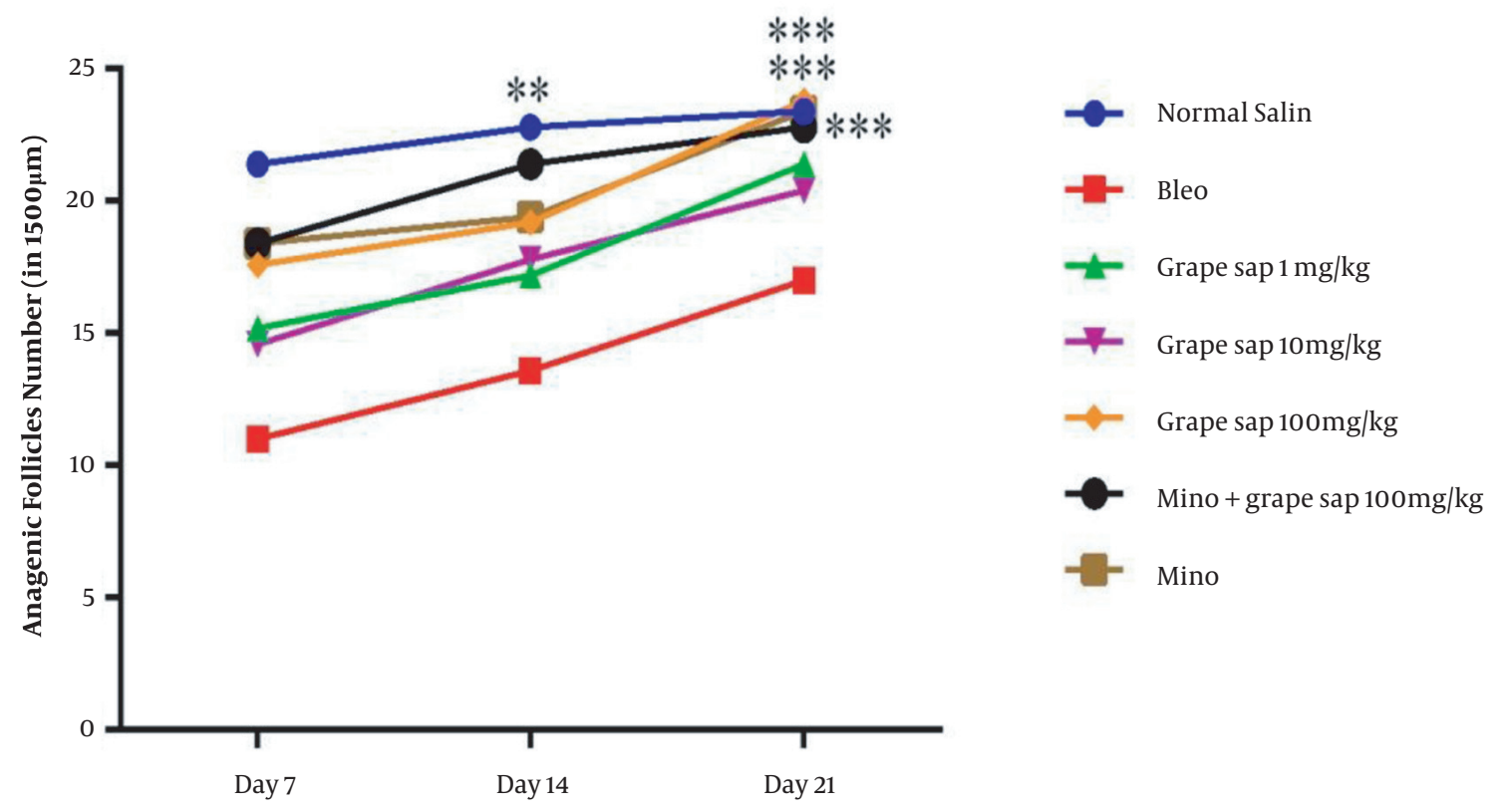

Figure 2. Mean number of hair follicles in $1500 \mu \mathrm{m}$ in experimental groups on days 7, 14, and 21; there were significant differences between the experimental groups and the bleomycin-treated group in terms of the number of hair follicles $(\mathrm{P}<0.001, \mathrm{P}<0.01$, and $\mathrm{P}<0.05)$. The results are presented as mean \pm standard error of the mean and evaluated using a two-way analysis of variance.

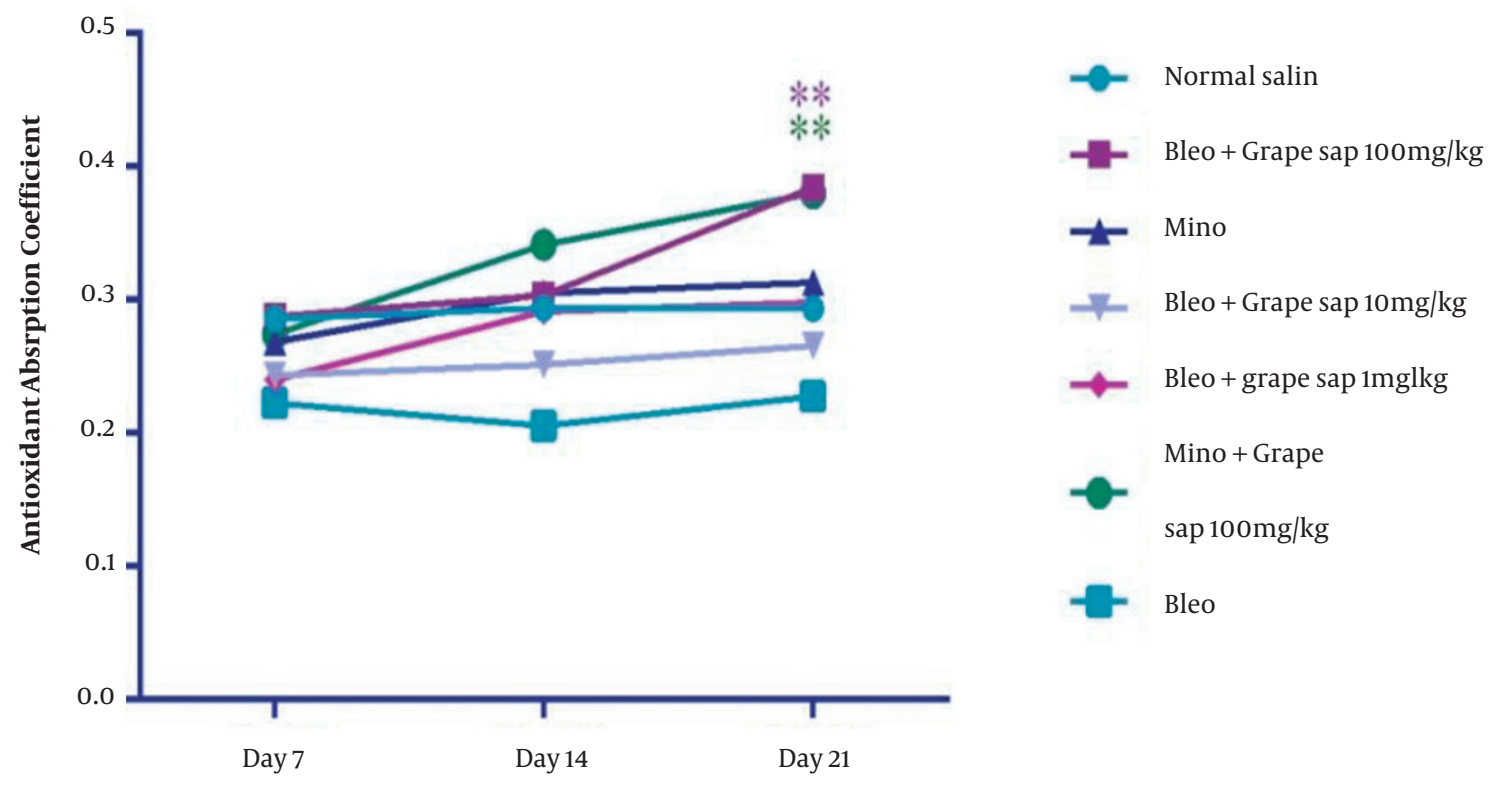

Figure 3. Total antioxidant capacity (antioxidant absorption coefficient) in experimental groups on days 7,14, and 21; there were significant differences between the experimental groups and the bleomycin-treated group in terms of total antioxidant capacity $(\mathrm{P}<0.001, \mathrm{P}<0.01$, and $\mathrm{P}<0.05)$. The results are presented as mean \pm standard error of the mean and evaluated using a two-way analysis of variance. 

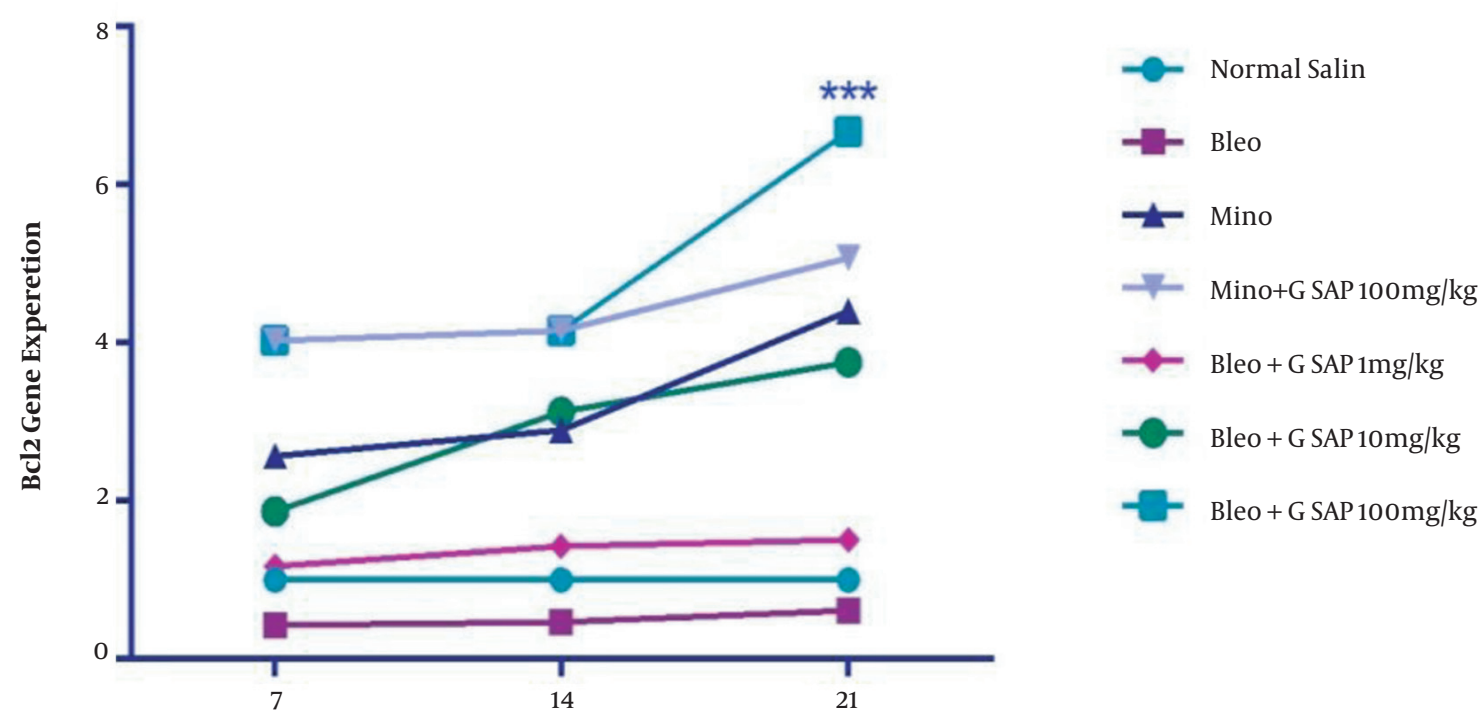

Day Test

Figure 4. Mean $B C L 2$ gene expression in experimental groups on days 7,14, and 21; there were significant differences between the grape sap-treated groups and the bleomycintreated group $(\mathrm{P}<0.001, \mathrm{P}<0.01$, and $\mathrm{P}<0.05)$. The results are presented as mean \pm standard error of the mean and evaluated using a two-way analysis of variance. With regard to the results of real-time polymerase chain reaction, a significant increase was observed in terms of $B A X$ expression.

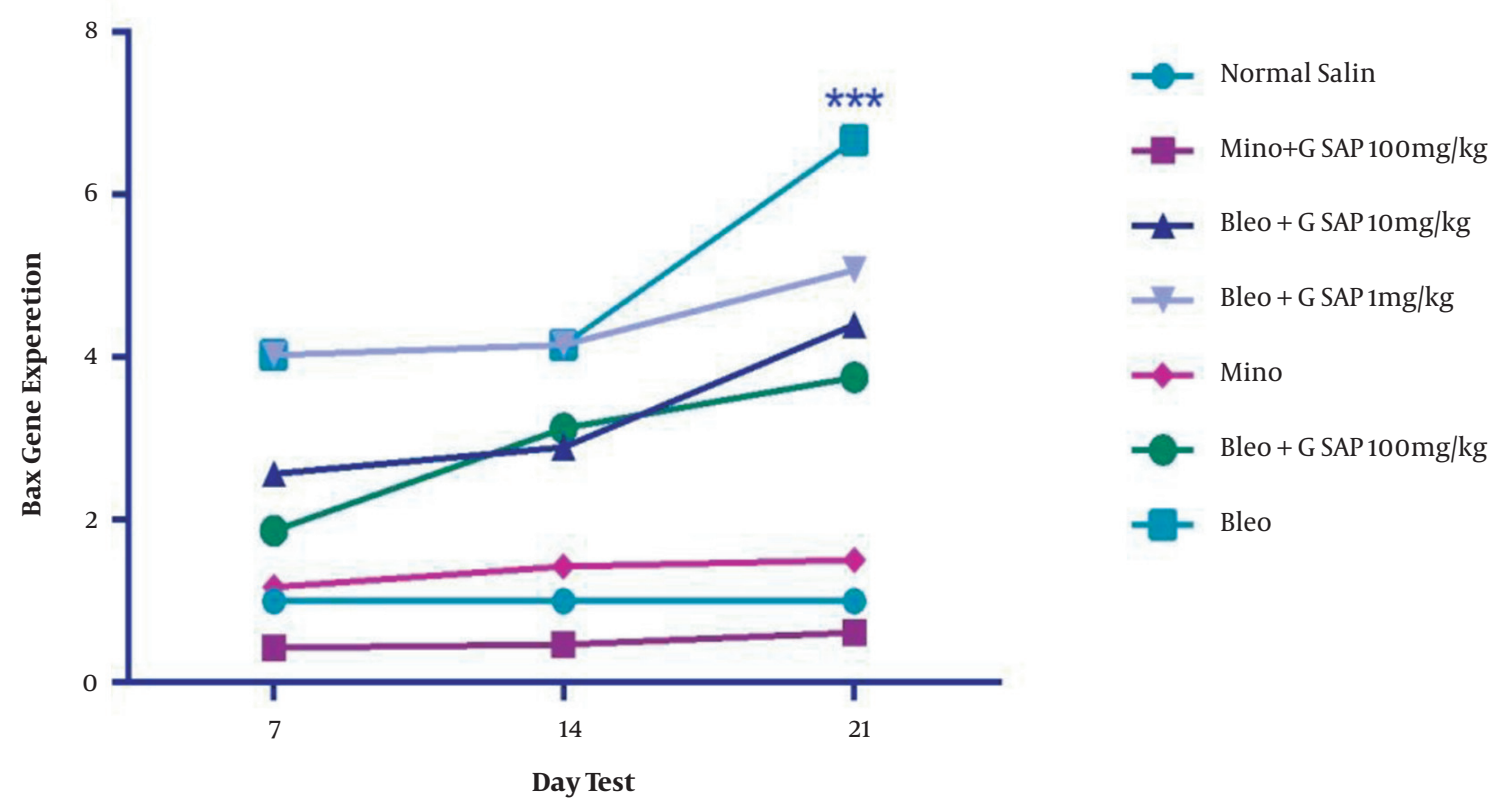

Figure 5. Mean $B A X$ gene expression in experimental groups on days 7,14, and 21; the results were significant in the bleomycin-treated group. There were significant differences between the experimental groups and the normal saline group in terms of mean $B A X$ expression $(\mathrm{P}<0.001, \mathrm{P}<0.01$, and $\mathrm{P}<0.05)$. The results are presented as mean \pm standard error of the mean and evaluated using a two-way analysis of variance. 
in anagenic follicles, which resulted from the presence of procyanidin. Kamimura et al. showed the in vivo and in vitro effect of grape seed proanthocyanidins on follicular cells. The results of the aforementioned study indicated that the grape seed increased the expression of genes associated with the growth, thereby leading to hair growth (28). These findings are not consistent with the findings of the present study.

In another study, Kazemi et al. demonstrated that the oral consumption of grape sap increases the number of follicles, which is consistent with the results of the current study (20). The genes involved in apoptosis rather than growth were investigated in study; however, both studies showed hair growth in the groups treated with grape seed extract and grape sap. According to the results, grape sap can be employed as a non-chemical drug due to its rich compounds, especially antioxidants. The results of recent studies indicated the positive effects of grape sap on the number of follicles and sebaceous glands, growth length, and blood vessel density at the base of every hair follicle. Moreover, grape sap decreases apoptosis in hair follicle cells by increasing the expression ratio of the $B C L 2 \mid B A X$, consequently stimulating hair growth.

\section{Acknowledgments}

This article was extracted from a master thesis in anatomy. The authors would like to express their gratitude to all individuals who helped with this study.

\section{Footnotes}

Authors' Contribution: Zahra Esmaeilzadeh, assistance in practical work; Ahmad Shabanizadeh, assistance in practical work; Zahra Taghipour, scientific advisor; Reza Vazirinejad, statistical consultant and English editor; Mohammad-Reza Salahshoor, consulting; Mohammad Mohsen Taghavi, designing the study protocol.

Conflict of Interests: The authors declare that there is no conflict of interest.

Ethical Approval: All applicable international, national, and institutional guidelines for the care and use of animals were followed in this study. This project was approved by the related Ethics Committee of Rafsanjan University of Medical Sciences (ethics code: IR.RUMS.REC.1396.55).

Funding/Support: This study was partly supported by a grant (no. 96047) from Rafsanjan University of Medical Sciences.

\section{References}

1. Van Der Donk J, Hunfeld JA, Passchier J, Knegt-Junk KJ, Nieboer C. Quality of life and maladjustment associated with hair loss in women with alopecia androgenetica. Soc Sci Med. 1994;38(1):159-63. doi: 10.1016/0277-9536(94)90311-5. [PubMed: 8146707].

2. Williamson D, Gonzalez M, Finlay AY. The effect of hair loss on quality of life. J Eur Acad Dermatol Venereol. 2001;15(2):137-9. doi: 10.1046/j.1468-3083.2001.00229.x. [PubMed: 11495520].

3. Price VH, Roberts JL, Hordinsky M, Olsen EA, Savin R, Bergfeld W, et al. Lack of efficacy of finasteride in postmenopausal women with androgenetic alopecia. J Am Acad Dermatol. 2000;43(5 Pt 1):768-76. doi: 10.1067/mjd.2000.107953. [PubMed: 11050579].

4. Katsarou-Katsari A, Singh LK, Theoharides TC. Alopecia areata and affected skin CRH receptor upregulation induced by acute emotional stress. Dermatology. 2001;203(2):157-61. doi: 10.1159/000051732. [PubMed: 11586016].

5. Shrivastava SB. Diffuse hair loss in an adult female: Approach to diagnosis and management. Indian J Dermatol Venereol Leprol. 2009;75(1):20-7. quiz 27-8. doi: 10.4103/0378-6323.45215. [PubMed: 19172026].

6. Masmoudi J, Sellami R, Ouali U, Mnif L, Feki I, Amouri M, et al. Quality of life in alopecia areata: A sample of tunisian patients. Dermatol Res Pract. 2013;2013:983804. doi: 10.1155/2013/983804. [PubMed: 23970896]. [PubMed Central: PMC3732641].

7. Botchkarev VA. Molecular mechanisms of chemotherapy-induced hair loss. J Investig Dermatol Symp Proc. 2003;8(1):72-5. doi: 10.1046/j.1523-1747.2003.12175.x. [PubMed: 12894998].

8. Lucky AW, Piacquadio DJ, Ditre CM, Dunlap F, Kantor I, Pandya AG, et al. A randomized, placebo-controlled trial of $5 \%$ and $2 \%$ topical minoxidil solutions in the treatment of female pattern hair loss. $J$ Am Acad Dermatol. 2004;50(4):541-53. doi: 10.1016/j.jaad.2003.06.014. [PubMed: 15034503].

9. Trusson D, Pilnick A. The role of hair loss in cancer identity: Perceptions of chemotherapy-induced alopecia among women treated for early-stage breast cancer or ductal carcinoma in situ. Cancer Nurs. 2017;40(2):E9-E16. doi: 10.1097/NCC.0000000000000373. [PubMed: 27070222].

10. Dubrey SW, VanGriethuysen J, Edwards CM. A hairy fall: syncope resulting from topical application of minoxidil.BMJ Case Rep.2015;2015. doi: 10.1136/bcr-2015-210945. [PubMed: 26347235]. [PubMed Central: PMC4567759].

11. Arca E, Acikgoz G, Tastan HB, Kose O, Kurumlu Z. An open, randomized, comparative study of oral finasteride and $5 \%$ topical minoxidil in male androgenetic alopecia. Dermatology. 2004;209(2):117-25. doi: 10.1159/000079595. [PubMed: 15316165].

12. Kaufman KD, Olsen EA, Whiting D, Savin R, DeVillez R, Bergfeld W, et al. Finasteride in the treatment of men with androgenetic alopecia.J $\mathrm{Am}$ Acad Dermatol.1998;39(4):578-89. doi: 10.1016/s0190-9622(98)70007-6.

13. Irwig MS, Kolukula S. Persistent sexual side effects of finasteride for male pattern hair loss. J Sex Med. 2011;8(6):1747-53. doi: 10.1111/j.17436109.2011.02255.x. [PubMed: 21418145].

14. Fong HH. Integration of herbal medicine into modern medical practices: Issues and prospects. Integr CancerTher. 2002;1(3):287-93. discussion 293. doi: 10.1177/153473540200100313. [PubMed: 14667286].

15. Ashrafi K, Esmaeli E, Fard S, Ansari Samani R, Parvin N, Namjoo A, et al. [The effect of hydroalcoholic extracts of Zizipus vulgaris L. on burn healing].J Shahrekord Univ Med Sci. 2011;12(4):78-82. Persian.

16. Leyden J, Dunlap F, Miller B, Winters P, Lebwohl M, Hecker D, et al. Finasteride in the treatment of men with frontal male pattern hair loss. J Am Acad Dermatol. 1999;40(6 Pt 1):930-7. doi: 10.1016/s01909622(99)70081-2. [PubMed: 10365924].

17. Libecco JF, Bergfeld WF. Finasteride in the treatment of alopecia. Expert Opin Pharmacother. 2004;5(4):933-40. doi: 10.1517/14656566.5.4.933. [PubMed: 15102575].

18. Nicolescu MP, Nicolescu R. Ocular sevatherapy with the help of crying sap from grape vine. AMT. 2012;2(1):165-8. 
19. Brzezinska-Wcislo L. [Study of the effect of vinblastine on hair growth in rats]. Przegl Dermatol. 1990;77(1):14-9. Polish. [PubMed: 2382011].

20. Kazemi M, Taghavi MM, Shabanizadeh A, Taghipour Z, Kaeidi A, Shariati-Kohbanani M. Investigation of grape sap on wingless/integrated and beta-catenin genes expression with histological factors on the hair follicle in rat. Res Pharm Sci. 2020;15(4):350-7. doi: 10.4103/1735-5362.293513. [PubMed: 33312213]. [PubMed Central: PMC7714015].

21. Yoon JI, Al-Reza SM, Kang SC. Hair growth promoting effect of Zizyphus jujuba essential oil. Food Chem Toxicol. 2010;48(5):1350-4. doi: 10.1016/j.fct.2010.02.036. [PubMed: 20206225].

22. Takahashi T, Kamiya T, Hasegawa A, Yokoo Y. Procyanidin oligomers selectively and intensively promote proliferation of mouse hair epithelial cells in vitro and activate hair follicle growth in vivo. J Invest Dermatol. 1999;112(3):310-6. doi: 10.1046/j.1523-1747.1999.00532.x. [PubMed: 10084307].

23. Li Z, Li J, Gu L, Begum S, Wang Y, Sun B, et al. Chrysanthemum zawadskii extract induces hair growth by stimulating the proliferation and differentiation of hair matrix. Int J Mol Med. 2014;34(1):130-6. doi: 10.3892/ijmm.2014.1768. [PubMed: 24807783].

24. Singha I, Das SK. Grapevine fruit extract protects against radiationinduced oxidative stress and apoptosis in human lymphocyte. Indian JExp Biol. 2015;53(11):753-61. [PubMed: 26669019].

25. Patel S, Sharma V, Chauhan NS, Thakur M, Dixit VK. Hair growth: Focus on herbal therapeutic agent. Curr Drug Discov Technol. 2015;12(1):21-42. doi: 10.2174/1570163812666150610115055. [PubMed: 26058803].

26. Koparal AT, Bostancioglu RB. Promotion of hair growth by traditionally used delphinium staphisagria seeds through inducti on of angiogenesis. Iran J Pharm Res. 2016;15(2):551-60. [PubMed: 27642326]. [PubMed Central: PMC5018283].

27. Zhong JY, Cong HQ, Zhang LH. Inhibitory effects of grape procyanidins on free radical-induced cell damage in rat hepatocytes in vitro. World J Gastroenterol. 2007;13(19):2752-5. doi: 10.3748/wjg.v13.119.2752. [PubMed: 17569148]. [PubMed Central: PMC4147128].

28. Kamimura A, Takahashi T. Procyanidin B-3, isolated from barley and identified as a hair-growth stimulant, has the potential to counteract inhibitory regulation by TGF-beta1. Exp Dermatol. 2002;11(6):53241. doi: 10.1034/j.1600-0625.2002.110606.x. [PubMed: 12473061]. 